\title{
Estudo comparativo do consumo de oxigênio e limiar anaeróbio em um teste de esforço progressivo entre atletas profissionais de futebol e futsal
}

Ernesto Cesar Pinto Leal J unior' ${ }^{1}$, Fabiano de Barros Souza², Márcio Magini ${ }^{3}$ e Rodrigo Álvaro Brandão Lopes Martins ${ }^{4}$

\section{RESUMO}

O consumo de oxigênio $\left(\mathrm{V}_{2}\right)$ tem sido de grande valia na avaliação funcional de atletas. A ergoespirometria é um procedimento não invasivo, utilizado para avaliar o desempenho físico ou a capacidade funcional de um indivíduo, conciliando a análise de gases espirados e variáveis respiratórias. No esporte, esse método de avaliação é de fundamental importância, pois traz significativa contribuição na verificação de índices de aptidão cardiorrespiratória, como é o caso do consumo máximo de oxigênio $\left(\mathrm{V}_{2}{ }_{2 a ́ x}\right)$ e o limiar anaeróbio (LA). O presente estudo teve como objetivo realizar uma comparação do consumo de oxigênio e limiar anaeróbio entre atletas profissionais de futebol e futsal em um teste progressivo. Participaram voluntariamente 31 indivíduos do sexo masculino, sendo: 19 atletas profissionais de futebol e 12 atletas profissionais de futsal. Os atletas foram submetidos a um protocolo de avaliação cardiorrespiratória progressiva pelo método ergoespirométrico e os resultados foram analisados quanto a sua diferença estatística através do teste $t$ de Student $(p<0,05)$. Os valores de $\mathrm{V}_{2}$ pico médios entre os dois grupos não apresentaram diferença estatisticamente significante ( $p>0,05$ ); entretanto, houve diferença estatística entre os dois grupos em relação ao limiar anaeróbio $(L A)(p<0,05)$. Com base nos resultados obtidos em nosso estudo pudemos concluir que, mesmo praticando esportes com diferentes características, os atletas de ambos os grupos possuem valores similares de consumo de oxigênio, porém, o limiar anaeróbio entre os dois grupos não apresentou a mesma similaridade, sugerido maior predominância do metabolismo anaeróbio durante o exercício nos atletas de futsal.

1. Mestre em Engenharia Biomédica. Doutorando em Ciências Cardiovasculares (UFRGS). Fisioterapeuta. Docente da Disciplina de Fisioterapia Desportiva do Centro Universitário La Salle (UNILASALLE), Canoas, RS. Docente da Disciplina de Monitoramento da Funcionalidade do Movimento Humano na Adolescência da Universidade de Caxias do Sul (UCS), Pesquisador do Laboratório do Movimento Humano (LMH) da Universidade de Caxias do Sul (UCS), Caxias do Sul, RS.

2. Mestre em Engenharia Biomédica. Educador Físico, Laboratório de Avaliação do Esforço Físico - LAEF, Universidade do Vale do Paraíba (UNIVAP), São J osé dos Campos, SP.

3. Doutor em Ciências. Físico, Laboratório de Matemática Aplicada, Instituto de Pesquisa e Desenvolvimento - IP\&D, Universidade do Vale do Paraíba (UNIVAP), São J osé dos Campos, SP.

4. Doutor em Biologia Celular e Molecular, Biólogo, Laboratório de Farmacologia e Fototerapia da Inflamação, Departamento de Farmacologia, Instituto de Ciências Biomédicas, Universidade de São Paulo (USP), São Paulo, SP.

Recebido em 1/11/05. Versão final recebida em 5/6/06. Aceito em 24/8/06.

Endereço para correspondência: Ernesto Cesar Pinto Leal J unior, Prof. MSc., FT, Rua Ernesto Alves, 1.919, apto. 74, Centro - 95020-360 - Caxias do Sul, RS. E-mail: ecplealj@ucs.br

\begin{abstract}
Palavras-chave: Análise comparativa. Fisiologia do esforço. Ergoespirometria. $\mathrm{VO}_{2}$. Capacidade aeróbia.

Keywords: $\quad$ Comparative analysis. Exertion physiology. Ergospirometry. $\dot{V}_{2}$ Aerobic ability.

Palabras-clave: Análisis comparativo. Fisiología del esfuerzo. Ergoespirometría. $\dot{V}_{2}$ Capacidad aeróbica.
\end{abstract}

\section{ABSTRACT \\ Comparative study of the oxygen consumption and anaerobic threshold in a progressive exertion test in professional soccer and indoor soccer athletes}

Oxygen consumption $\left(\mathrm{VO}_{2}\right)$ has been very useful for the functional evaluation of athletes. Ergospirometry is a non-invasive procedure used to evaluate the physical performance or the functional ability of an individual, connecting the analysis of the inspired gases with the respiratory variables. This evaluation method is extremely important to sports, since it brings significant contribution in the verification of indices of cardiorespiratory aptitude, which is the case $f$ the maximal oxygen consumption $\left(\mathrm{VO}_{2 \max }\right)$ and the anaerobic threshold (AT). The present study had as objective to compare oxygen consumption and anaerobic threshold in professional soccer and indoor soccer athletes in a progressive test. 31 male individuals voluntarily participated in the test, being: 19 professional soccer athletes and 12 professional indoor soccer athletes. The athletes were submitted to a progressive cardiorespiratory evaluation protocol through the ergospirometric method, and the results were analyzed conceming their statistical difference through the $t$-Student test $(p<0,05)$. The average $\mathrm{V}_{2 \text { peak }}$ indices between the two groups did not present statistically significant difference $(p>0,05)$; however, there was statistically difference between the two groups concerning the anaerobic threshold (AT) $(p<0,05)$. Based on the results obtained in our study we concluded that even practicing sports with different characteristics, the athletes from both groups have similar indices of oxygen consumption. Nevertheless, the anaerobic threshold between the two groups did not present the same similarity, suggesting higher predominance of anaerobic metabolism during exercise in the indoor soccer athletes.

\section{RESUMEN}

\section{Estudio comparativo del consumo de oxígeno y límite anaeróbico en un test de esfuerzo progresivo entre atletas profesionales de fútbol y fútbol de salón}

El consumo de oxígeno $\left(\mathrm{VO}_{2}\right)$ ha sido de gran valía en la evaluación funcional de atletas. La ergoespirometría es un procedimiento no invasivo, utilizado para evaluar el desempeño físico o la capacidad funcional de un individuo, conciliando el análisis de gases expirados y las variables respiratorias. En el deporte, ese método 
de evaluación es de fundamental importancia, pues contribuye significativamente en la verificación de índices de aptitud cardiorrespiratoria, como es el caso del consumo máximo de oxígeno $\mathrm{V}_{\mathrm{V}} \mathrm{O}_{2}$ máx.) y el límite anaeróbico (LA). El presente estudio ha tenido como objetivo realizar una comparación del consumo de oxígeno y límite anaeróbico entre atletas profesionales de fútbol y fútbol de salón en un test progresivo. Participaron voluntariamente 31 individuos del sexo masculino, entre ellos: 19 atletas profesionales de fútbol, y 12 atletas profesionales de fútbol de salón. Los deportistas fueron sometidos a un protocolo de evaluación cardiorrespiratoria progresiva por el método ergoespirométrico, y os resultados fueron analizados en relación a su diferencia estadística a través del test t-Student $(p<0,05)$. Los valores de $\dot{V}_{2}$ pico medios entre los dos grupos no presentaron diferencia estadistica significante ( $p>0,05)$; a pesar de eso hubo diferencia estadística entre los dos grupos en relación al límite anaeróbico $(L A)(p<0,05)$. Teniendo como base los resultados obtenidos en nuestro estudio pudimos concluir que incluso practicando deportes con diferentes características, los atletas de ambos grupos poseen valores similares de consumo de oxígeno, sin embargo el límite anaeróbico entre los dos grupos no presentó la misma similitud, sugiriendo mayor predominancia del metabolismo anaeróbico durante el ejercicio en los atletas de fútbol de salón.

\section{INTRODUÇÃO}

O consumo máximo de oxigênio $\left(\dot{\mathrm{V}}_{2}\right.$ máx. $)$ pode ser definido como o maior volume de oxigênio por unidade de tempo que um indivíduo consegue captar, respirando ar atmosférico durante o exercício. É alcançado quando se atingem níveis máximos de débito cardíaco e de extração periférica de oxigênio e não é ultrapassado mesmo com incremento na carga de trabalho muscular(1).

$\mathrm{O} \mathrm{V}_{2}$ máx. vem sendo considerado um dos parâmetros de grande importância como preditor de performance, pois a capacidade do ser humano para realizar exercícios de longa e média duração depende principalmente do metabolismo aeróbio sendo, assim, um índice muito empregado para classificar a capacidade funcional cardiorrespiratória, sobretudo em atletas ${ }^{(2-3)}$.

O limiar anaeróbio é a transição do metabolismo aeróbio para o anaeróbio e é também um índice que reflete satisfatoriamente a aptidão física, sendo empregado tanto na prática clínica como na avaliação e no treinamento de atletas ${ }^{(4-5)}$.

O limiar anaeróbio, que quando caracterizado exclusivamente em função das trocas respiratónias recebe a denominação de "limiar ventilatório", pode ser definido como a intensidade de esforço acima da qual a produção de ácido láctico supera sua própria remoção, provocando hiperventilação(6).

A ergoespirometria é um procedimento não invasivo, utilizado para avaliar o desempenho físico ou a capacidade funcional de um indivíduo, conciliando a análise de gases espirados, variáveis respiratórias e oximetria(7).

Esse método tem sido útil na determinação de fatores ligados a indicadores preditores de performance, identificação de intolerância ao exercício, determinantes de transição metabólica, avaliação clínica e terapêutica de diversas patologias, prescrição de intensidade do exercício, índices de eficiência respiratónia e cardiovascular e custo energético ${ }^{(2,8)}$.

Diversos estudos foram realizados com o objetivo de avaliar o condicionamento físico de atletas de futebol. Entretanto, existe escassez de estudos com o mesmo objetivo envolvendo atletas de futsal.

Por serem duas modalidades similares em seus gestos esportivos, porém com grande discrepância em relação às dimensões físicas em que são praticadas, indaga-se quais seriam também as semelhanças e diferenças encontradas nas variáveis respiratórias e no metabolismo de seus praticantes.
O presente estudo teve como objetivo avaliar o condicionamento físico de atletas de futebol e futsal, bem como comparar o consumo de oxigênio $\left(\mathrm{VO}_{2}\right)$ e o limiar anaeróbio (LA) desses grupos de atletas.

\section{METODOLOGIA}

Foram estudados 31 indivíduos do sexo masculino, atletas, divididos em dois grupos distintos:

Grupo futebol: composto por 19 atletas profissionais de futebol da equipe do Esporte Clube Taubaté, com idade média de 24,3 anos $( \pm 2,3)$, estatura média de $178,8 \mathrm{~cm}( \pm 6,3)$ e massa corporal média de 75,1kg ( $\pm 7,5)$.

Grupo futsal: composto por 12 atletas profissionais de futsal da equipe da Assem/FADENP de São J osé dos Campos, com idade média de 20,9 anos $( \pm 2,7)$, estatura média de $176,0 \mathrm{~cm}( \pm 6,0)$ e massa corporal média de $69,9 \mathrm{~kg}( \pm 5,9)$.

Participaram deste estudo os indivíduos que preencheram os seguintes critérios de inclusão: a) não apresentem histórico de pneumopatias nos últimos 12 meses; b) não apresentem cardiopatias; c) realizem treinamento com freqüência mínima de cinco vezes semanais e quatro horas por dia.

O estudo foi submetido e aprovado pelo Comitê de Ética e Pesquisa (CEP) da Universidade do Vale do Paraíba-UNIVAP. Os indivíduos foram informados deste estudo e foi aplicado temo de consentimento, conforme determina a resolução 196/96 do CNS.

Foi utilizado para o estudo um eletrocardiógrafo digital Micromed para registro e análise do ECG durante o esforço; uma esteira ergométrica marca Inbrasport, modelo Super ATL; um analisador de gases VO 2000 da marca MedGraphics, acoplado a um microcomputador, equipado com o software Elite produzido pela M icromed; clipe nasal; eletrodos descartáveis; lâminas de barbear descartáveis; lixas descartáveis; álcool 70.

Os testes foram realizados na pré-temporada dos dois grupos de atletas estudados no Laboratório de Avaliação do Esforço Físico-LAEF, na Faculdade de Ciências da Saúde-FCS da Universidade do Vale do Paraíba-UNIVAP.

A execução dos testes ocorreu em um ambiente controlado, a uma temperatura ambiente de $24^{\circ} \mathrm{C}$, e com umidade relativa do ar de $60 \%$.

O protocolo de avaliação utilizado foi de esforço progressivo, com inclinação constante de $3 \%$ e velocidade inicial de $4,0 \mathrm{~km} / \mathrm{h}$. Houve incremento de velocidade de $1,0 \mathrm{~km} / \mathrm{h}$ a cada minuto de teste até o quarto minuto; posteriormente ao quarto minuto o incremento de velocidade também foi de $1,0 \mathrm{~km} / \mathrm{h}$, porém ocorrendo a cada dois minutos de teste.

A duração dos testes foi determinada pela exaustão do atleta, que quando o atingia sinalizava ao avaliador, que iniciava então a fase de recuperação com velocidade inicial de 5,0km/h, com decremento de $1,0 \mathrm{~km} / \mathrm{h}$ após o primeiro minuto mantido até o final da recuperação, determinada pela estabilização da freqüência cardíaca do atleta.

Os dados obtidos foram posteriormente comparados entre os dois grupos através de sua média aritmética e analisados quanto a sua diferença estatística através do teste $t$ de Student não-pareado, com nível de significância de $p<0,05$.

\section{RESULTADOS}

No grupo estudado de atletas profissionais de futebol, o $\mathrm{V}_{2}$ pico médio foi de $4,20 \mathrm{~L} / \mathrm{min}( \pm 0,31$ ) em valores absolutos e $54,8 \mathrm{ml} /$ $\mathrm{kg} / \mathrm{min}( \pm 4,02)$ em valores relativos à massa corporal; o limiar anaeróbio respiratório médio do grupo ocorreu com um consumo de oxigênio de $3,46 \mathrm{~L} / \mathrm{min}( \pm 0,35)$, com 14 minutos de teste ( \pm 1,67).

J á no grupo de atletas profissionais de futsal, o $\mathrm{VO}_{2}$ pico médio foi de $3,89 \mathrm{~L} / \mathrm{min}( \pm 0,43)$ em valores absolutos e $55,7 \mathrm{ml} / \mathrm{kg} / \mathrm{min}$ 
$( \pm 3,70)$ em valores relativos à massa corporal; o limiar anaeróbio respiratório médio do grupo ocorreu com um consumo de oxigênio de $2,97 \mathrm{~L} / \mathrm{min}( \pm 0,44)$, com 11 minutos e 40 segundos de teste $( \pm 1,33)$ (tabela 1$)$.

\begin{tabular}{|c|c|c|c|}
\hline \multicolumn{4}{|c|}{$\begin{array}{c}\text { TABELA } 1 \\
\text { Consumo de oxigênio pico e limiar anaeróbio - } \\
\text { grupo futebol e grupo futsal }\end{array}$} \\
\hline & Futebol & Futsal & $\begin{array}{l}\text { Significância } \\
\text { estatística }\end{array}$ \\
\hline$\dot{\mathrm{V}} \mathrm{O}_{2 \text { pico }}(\mathrm{L} / \mathrm{min})$ & $4,20( \pm 0,31)$ & $3,89( \pm 0,43)$ & $p>0,05$ \\
\hline Limiar anaeróbio (L/min) & $3,46( \pm 0,35)$ & $2,97( \pm 0,44)$ & $p<0,05^{*}$ \\
\hline
\end{tabular}

* Estatisticamente significante.

Para melhor visualização dos dados, o gráfico 1 mostra as curvas médias de consumo de oxigênio $\left(\mathrm{V}_{2}\right)$ e limiar anaeróbio (LA) obtidos nos dois grupos estudados.

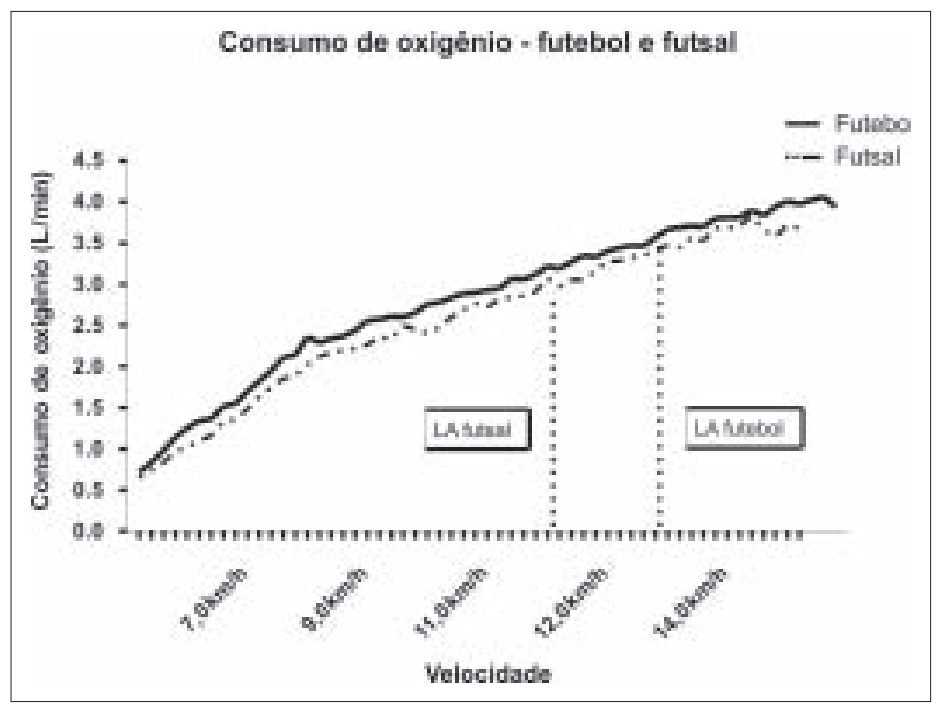

Gráfico 1 - Consumo de oxigênio $\left(\mathrm{VO}_{2}\right)$ dos dois grupos estudados

\section{DISCUSSÃO}

Os valores obtidos do pico de consumo de oxigênio $\left(\mathrm{V}_{2}\right.$ pico $)$ entre os dois grupos estudados: futebol - 4,20L/min em valores absolutos e $54,8 \mathrm{ml} / \mathrm{kg} / \mathrm{min}$ em valores relativos à massa corporal; futsal $-3,89 \mathrm{~L} / \mathrm{min}( \pm 0,43)$ em valores absolutos e $55,7 \mathrm{ml} / \mathrm{kg} / \mathrm{min}$ $( \pm 3,70)$ em valores relativos à massa corporal, não apresentaram diferença estatisticamente significante $(p>0,05)$.

Nossos dados se aproximam aos de Diaz ${ }^{(9)}$, que realizou um estudo cujo objetivo foi examinar as tendências das variáveis cardiovasculares e metabólicas em 248 jogadores profissionais de futebol de elite em um período de 27 anos (1973-2000). Os valores do consumo máximo de oxigênio foram de $54 \mathrm{ml} / \mathrm{kg} / \mathrm{min}$ na década de 1980 e $57 \mathrm{ml} / \mathrm{kg} / \mathrm{min}$ na década de 1990 .

Al-Hazzaa et al.(10) realizaram uma avaliação cardiorrespiratória com 23 jogadores da seleção saudita de futebol, em que os parâmetros cardiorrespiratórios foram avaliados com o método ergoespirométrico de circuito aberto. Os valores médios obtidos do $\mathrm{VO}_{2 \text { máx. }}$ absoluto e relativo à massa corporal foram, respectivamente, de $4,16 \mathrm{~L} / \mathrm{min}$ e $56,8 \mathrm{ml} / \mathrm{kg} / \mathrm{min}$, sendo similares aos de nosso estudo. Segundo os autores, o $\mathrm{V}_{2}$ máx. relativo à massa corporal é menor do que os valores relatados na literatura de acordo com a modalidade, porém, tal fato se explica pela realização dos testes ter ocorrido na pré-temporada.

Entretanto, esses valores são diferentes dos encontrados por Tumiltyy ${ }^{(11)}$, para quem os atletas de futebol possuem em média um consumo máximo de oxigênio de $60 \mathrm{ml} / \mathrm{kg} / \mathrm{min}$.
Os valores do nosso estudo também diferem dos obtidos por Raven et al.(12), que realizaram um estudo com a finalidade de avaliar quanto à sua função fisiológica uma equipe profissional de futebol da liga norte-americana de futebol (NASL). Dezoito jogadores foram avaliados quanto à sua função cardiorrespiratória, resistência ao exercício, composição corporal e tiveram mensuradas suas aptidões motoras no final da temporada. O consumo máximo de oxigênio $\left(\mathrm{V}_{2} \mathrm{máx}_{\text {. }}\right)$ foi de $58,4 \mathrm{ml} / \mathrm{kg} / \mathrm{min}$.

Com o objetivo de comparar o consumo de oxigênio máximo $\left(\mathrm{V}_{2}\right.$ máx. $)$ em um teste de campo específico para futebol e um teste ergoespirométrico convencional, Kemi et al.(13) obtiveram valores similares de $\dot{\mathrm{V}}_{2 \text { máx }}$ entre os testes específicos realizados em campo (5,0L/min) e a ergoespirometria em laboratório (5,1L/ $\mathrm{min})$, diferentemente dos resultados encontrados em nosso estudo.

Nossos resultados também são diferentes dos obtidos por Casajus ${ }^{(14)}$, em uma equipe profissional espanhola de futebol com 15 atletas no início e ao final da temporada. Quanto ao consumo máximo de oxigênio, o autor observou não haver diferença significativa entre os testes realizados no início da temporada $(65,5 \mathrm{ml} /$ $\mathrm{kg} / \mathrm{min}$ ) e ao final da mesma $(66,4 \mathrm{ml} / \mathrm{kg} / \mathrm{min})$.

Chin et al. ${ }^{(15)}$ relataram um consumo de oxigênio de $59,1 \mathrm{ml} / \mathrm{kg} /$ min em 24 atletas profissionais de futebol de Hong Kong, resultados estes também diferentes dos obtidos em nosso estudo.

Nossos valores também diferem dos de Silva et al.(16), que avaliaram 18 jogadores de futebol profissional, os quais foram submetidos a um teste máximo em esteira ergométrica, utilizando-se um protocolo escalonado e contínuo, obtendo $\mathrm{V}_{2}$ pico de $63,75 \mathrm{ml} /$ $\mathrm{kg} / \mathrm{min}( \pm 4,93)$.

O grupo estudado de atletas de futsal atingiu o limiar anaeróbio (LA) em menor tempo em relação ao grupo estudado de atletas de futebol, sendo esta diferença estatisticamente significante ( $p$ $<0,05)$, mesmo tendo os dois grupos estudados valores similares de $\mathrm{VO}_{2}$. Tal fato poderia sugerir-nos maior predominância aeróbia no futebol quando comparado com o futsal(17).

Entretanto, os atletas de futsal permaneceram maior tempo no exercício anaeróbio em relação ao grupo de atletas de futebol. Tal fato poderia ser explicado pelo melhor condicionamento anaeróbio desses atletas, por menor compressão vascular local exercida pelos músculos durante a atividade, determinada por otimização do recrutamento de unidades motoras no metabolismo anaeróbio, ocasionada pela adaptação ao treinamento da modalidade ${ }^{18-}$ 19). Isso facilitaria a remoção do ácido láctico pelas vias circulatórias e, conseqüentemente, diminuiria a segunda fonte de produção de $\mathrm{CO}_{2}$ que ocorre acima do limiar anaeróbio, chamada de $\mathrm{CO}_{2}$ não metabólico, resultante do tamponamento do lactato, em níveis mais elevados de exercício(3,20).

Outro fato que poderia explicar a maior permanência dos atletas de futsal no exercício anaeróbio seria o mecanismo de "economia de corrida", no qual o indivíduo tem um gasto energético otimizado, que ocorreria em função do tipo de treinamento ao qual estes atletas são submetidos, que se assemelha a um treinamento intervalado(19,21-22).

\section{CONCLUSÃO}

Analisando os resultados obtidos, pudemos observar que os atletas avaliados nos dois grupos possuem capacidade aeróbia condizente com a relatada na literatura para atletas na pré-temporada.

Em valores absolutos e relativos, os dois grupos assemelhamse nos valores de consumo de oxigênio pico. Contudo, os atletas de futebol alcançaram o limiar anaeróbio mais tardiamente em relação aos atletas de futsal.

Entretanto, os atletas de futsal, apesar de atingirem o limiar anaeróbio de maneira mais precoce, permaneceram no metabolismo anaeróbio por mais tempo que o grupo estudado de atletas de futebol. Isso nos sugere predominância da atividade anaeróbia 
no futsal em relação ao futebol e, conseqüentemente, maior adaptação por parte desses atletas ao exercício anaeróbio, bem como melhor adaptação dos atletas de futebol ao exercício aeróbio.

Propõe-se que estudos futuros sejam realizados com a finalidade de analisar matematicamente o comportamento das curvas estudadas, bem como a influência do limiar anaeróbio no comportamento das mesmas, a fim de elucidar as diferenças evidenciadas no presente trabalho.

Todos os autores declararam não haver qualquer potencial conflito de interesses referente a este artigo.

\section{REFERÊNCIAS}

1. Taylor HL, Buskirk E, Henschel A. Maximal oxygen intake as an objective measure of cardiorespiratory performance. J Appl Physiol. 1955;8:73-80.

2. Silva PRS, Romano A, Yazbek J r P, Cordeiro J R, Battistella LR. Ergoespirometria computadorizada ou calorimetria indireta: um método não invasivo de crescente valorização na avaliação cardiorrespiratória ao exercício. Rev Bras Med Esporte. 1998;4:147-58.

3. Barros Neto TL, Tebexreni AS, Tambeiro VL. Aplicações práticas da ergoespirometria no atleta. Rev Soc Cardiol Estado de São Paulo. 2001;11:695-705.

4. Barros Neto TL, Russo AK, Da Silva AC, Picarro IC, Griggio MA, Tarasantchi J . Potassium-induced ventilatory reflexes originating from the dog hindlimb during rest and passive exercise. Braz J Med Biol Res. 1981;14:285-90.

5. Hollmann W. Historical remarks on the development of the aerobic-anaerobic threshold up to 1966. Int J Sports Med. 1985;6:109-16.

6. Wassermann K, Mcllroy MB. Detecting the threshold of anaerobic metabolism in cardiac patients during exercise. Am J Cardiol. 1964;14:844-52.

7. Serra S. Considerações sobre ergoespirometria. Arq Bras Cardiol. 1997;68:3014.
8. Denis C, Dozmois D, Lacour J R. Endurance training, $\dot{V}_{2}$ max., and OBLA: a longitudinal study of two different groups. Int J Sports Med. 1984;5:167-73.

9. Diaz FJ . Changes of physical and functional characteristics in soccer players. Rev Invest Clin. 2003;55:528-34.

10. Al-Hazzaa HM, Almuzaini KS, Al-Refaee SA, Sulaiman MA, Dafterdar MY, Al-Ghamedi A, et al. Aerobic and anaerobic power characteristics of Saudi elite soccer players. J Sports Med Phys Fitness. 2001;41:54-61.

11. Tumilty D. Physiological characteristics of elite soccer players. Sports Med. 1993; 16:80-96.

12. Raven PB, Gettman LR, Pollock ML, Cooper KH. A physiological evaluation of professional soccer players. BrJ Sports Med. 1976;10:209-16.

13. Kemi OJ , Hoff J , Engen LC, Helgerud J, Wisloff U. Soccer specific testing of maximal oxygen uptake. J Sports Med Phys Fitness. 2003;43:139-44.

14. Casajus J A. Seasonal variation in fitness variables in professional soccer players. J Sports Med Phys Fitness. 2001;41:463-9.

15. Chin MK, Lo YS, Li CT, So CH. Physiological profiles of Hong Kong elite soccer players. BrJ Sports Med. 1992;26:262-6.

16. Silva PRS, Romano A, Teixeira AAA, Visconti AM, Roxo CDMN, Machado GS, et al. A importância do limiar anaeróbico e do consumo máximo de oxigênio $\left(\mathrm{V}_{2}\right.$ máx.) em jogadores de futebol. Rev Bras Med Esporte. 1999;5:225-32.

17. McArdle WD, Katch FI, Katch VL. Fundamentos de fisiologia do exercício. $2^{\text {nd }}$ ed. Rio de J aneiro: Guanabara Koogan, 2002.

18. Behm DG, Sale DG. Velocity specificity of resistance training. Sports Med. 1993; 15:374-88.

19. Stolen T, Chamari K, Castagna C, Wisloff U. Physiology of soccer: an update. Sports Med. 2005;35:501-36.

20. Beaver WL, Wasserman K, Whipp BJ . On-line computer analysis and breath-bybreath graphical display of exercise function tests. J Appl Physiol. 1973;34:12832.

21. Helgerud J , Engen LC, Wisloff U, Hoff J . Aerobic endurance training improves soccer performance. Med Sci Sports Exerc. 2001;33:1923-31.

22. Chamari K, Hachana Y, Kouach F, J eddi R, Moussa-Chamari I, Wisloff U. Endurance training and testing with the ball in young elite soccer players. Br J Sports Med. 2005;39:24-8. 